\title{
Characterization of Amino Acid Based Molecular Micelles with Molecular Modeling
}

\author{
Alexander Billiot ${ }^{1,2}$, Yayin Fang2*, Kevin F. Morris ${ }^{3}$ \\ ${ }^{1}$ Department of Physical and Environmental Sciences, Texas A \& M University-Corpus Christi, \\ Corpus Christi, TX, USA \\ ${ }^{2}$ Department of Biochemistry and Molecular Biology, College of Medicine, Howard University, Washington, DC, USA \\ ${ }^{3}$ Department of Chemistry, Carthage College, Kenosha, WI, USA \\ Email: *yfang@howard.edu
}

How to cite this paper: Billiot, A., Fang, Y.Y. and Morris, K.F. (2019) Characterization of Amino Acid Based Molecular Micelles with Molecular Modeling. Open Journal of Physical Chemistry, 9, 221-240. https://doi.org/10.4236/ojpc.2019.94014

Received: October 26, 2019

Accepted: November 26, 2019

Published: November 29, 2019

Copyright (๑) 2019 by author(s) and Scientific Research Publishing Inc. This work is licensed under the Creative Commons Attribution International License (CC BY 4.0).

http://creativecommons.org/licenses/by/4.0/

cc) (i) Open Access

\begin{abstract}
The enantiomers of chiral drugs often have different potencies, toxicities, and biochemical properties. Therefore, the FDA and other worldwide regulatory agencies require manufactures to test and prove the enantiomeric purity of chiral drugs. Amino acid based molecular micelles (AABMM) have been used in chiral CE separations since the 1990's because of their low environmental impact and because their properties can easily be tuned by changing the amino acids in the chiral surfactant head groups. Using molecular dynamics simulations to investigate the structures and properties of AABMM is part of an ongoing study focusing on investigating and elucidating the factors responsible for chiral recognition with AABMM. The results will be useful for the proper design and selection of more efficient chiral selectors. The micelles investigated contained approximately twenty covalently linked surfactant monomers. Each monomer was in turn composed of an undecyl hydrocarbon chain bound to a dipeptide headgroup containing of all combinations of L-Alanine, L-Valine, and L-Leucine. These materials are of interest because they are effective chiral selectors in capillary electrophoresis separations. Molecular dynamics simulation analyses were used to investigate how the sizes and positions of the headgroup amino acid R-groups affected the solvent accessible surface areas of each AABMM chiral center. In addition, headgroup dihedral angle analyses were used to investigate how amino acid R-group size and position affected the overall headgroup conformations. Finally, distance measurements were used to study the structural and conformational flexibilities of each AABMM headgroup. All analyses were performed in the context of a broader study focused on developing structure-based predictive tools to identify the factors responsible for a) self-assembly, b) function, c) higher ordered structure and d) molecular recognition of these amino acid based mo-
\end{abstract}


lecular micelles.

\section{Keywords}

Amino Acid Based Molecular Micelles, Molecular Modeling, Computational Chemistry

\section{Introduction}

The different physiological and pharmacological properties of chiral drug enantiomers often lead to many unforeseen consequences and side effects. One example is the chiral drug methamphetamine, where L-methamphetamine is a rarely prescribed nasal decongestant and D-methamphetamine is a well-known and highly illegal and addictive narcotic most commonly referred to as meth. Many techniques such as gas and liquid chromatography [1]-[9] and capillary electrophoresis (CE) [10]-[19] have been developed to separate the enantiomers of chiral compounds. These separations are used to analyze the results of chiral drug syntheses and to confirm the enantiomeric purity of commercial drug formulations. Chiral CE separations, when compared to other techniques, often have a lower operation cost, smaller sample size requirements, shorter analysis times, and higher separation efficiencies. In a 2019 review, Yu, et al. discussed several classes of chiral selectors used in CE separations. These included antibiotics like streptomycin, doxycycline, and vancomycin; polysaccharides such as glycosaminoglycans and maltodextrin as well as natural and derivatized cyclodextrins; chiral ionic liquids; oligonucleotides; and molecular micelles [20].

The class of CE selectors investigated here is amino acid based molecular micelles (AABMM). These selectors have been used in chiral CE separations since the 1990's because of their low environmental impact and because their properties can easily be tuned by changing the amino acids in the chiral surfactant headgroups. In AABMM, the surfactant monomers are polymerized via gamma radiation to increase their structural stability. Furthermore, covalently bound surfactants are in micellar form at all concentrations, whereas conventional surfactants only form micelles above their CMC (critical micelle concentration). This property allows separations to be carried out at lower concentrations often leading to improvements in chiral selectivity [13]-[19].

A significant challenge in these chiral CE separations is to identify the AABMM system and dipeptide headgroup that is likely to provide the most efficient and highest resolution analysis. Therefore, the long-term goal of this research is to build a database of quantitative structure-enantioselective retention relationships (QSERR) that can be used to evaluate and compare the effectiveness of different AABMM's in CE separation experiments. Analysts can then use these in silico tools to determine the best AABMM system for a given separation problem.

Here, we describe molecular dynamics simulation work undertaken to better 
characterize the structural and dynamic properties of a nine different AABMM systems. Micelle properties investigated included the water accessibility of the headgroup chiral centers and both the conformations and conformational flexibilities of each dipeptide headgroup. Insights from these MD simulation results will then be used to build the predictive models described above. We also note that since enantio-recognition in these systems is amino acid based, knowledge gained from these studies may be partially transferable to proteins and other amino acid based chiral selectors.

\section{Experimental Details}

The AABMMs examined in the study are shown in Table 1 . They include the undecyl forms of all dipeptide combinations of L-Alanine (A), L-Valine (V) and L-Leucine (L). Furthermore, since each micelle's counterion was sodium, the first two abbreviations shown in the first column in Table 1, represent sodium (S) and undecyl (U), respectively, while the last two letters refer to the amino acids making up the dipeptide headgroup. The number of repeat units listed in Table 1 is experimentally derived aggregation numbers for molecular micelles formed when 100 $\mathrm{mM}$ solutions containing the respective monomeric surfactants were exposed to gamma radiation [20]. When constructing the AABMM systems for MD simulation analysis, surfactant monomer chains were connected with covalent bonds at the end of each monomer's hydrocarbon tail. The method used was consistent with our previous work and is illustrated in Figure 1(a) [21]-[27]. Figure 1(b) shows a representative structure extracted from the SULL MD simulation. Note that as expected the SULL system formed a globular micellar structure.

Our previous MD simulation work focused on the AABMM SULL, SULV, and SUVL. Initial studies characterized the structural and dynamic properties of these micellar systems while subsequent work investigated chiral ligand association with each AABMM [21]-[27]. Throughout these studies, MD simulation

Table 1. Amino acid side chains and micelle aggregation numbers for each AABMM. R1 and $\mathrm{R} 2$ refer to the $\mathrm{N}$-terminal and $\mathrm{C}$-terminal amino acids, respectively.

\begin{tabular}{cccc}
\hline Surfactant & $\mathbf{R} 1$ & $\mathbf{R} 2$ & \# of Repeat Units \\
\hline SUAA & $\mathrm{CH}_{3}$ & $\mathrm{CH}_{3}$ & 26 \\
SUAV & $\mathrm{CH}_{3}$ & $\mathrm{CH}\left(\mathrm{CH}_{3}\right)_{2}$ & 24 \\
SUAL & $\mathrm{CH}_{3}$ & $\mathrm{CH}_{2} \mathrm{CH}\left(\mathrm{CH}_{3}\right)_{2}$ & 25 \\
SUVA & $\mathrm{CH}\left(\mathrm{CH}_{3}\right)_{2}$ & $\mathrm{CH}_{3}$ & 19 \\
SUVV & $\mathrm{CH}\left(\mathrm{CH}_{3}\right)_{2}$ & $\mathrm{CH}\left(\mathrm{CH}_{3}\right)_{2}$ & 23 \\
SUVL & $\mathrm{CH}\left(\mathrm{CH}_{3}\right)_{2}$ & $\mathrm{CH}_{2} \mathrm{CH}\left(\mathrm{CH}_{3}\right)_{2}$ & 19 \\
SULA & $\mathrm{CH}_{3}$ & $\mathrm{CH}_{3}$ & 18 \\
SULV & $\mathrm{CH}_{3}\left(\mathrm{CH}_{3}\right)_{2}$ & $\mathrm{CH}_{2}\left(\mathrm{CH}_{3}\right)_{2}$ & 18 \\
SULL & $\mathrm{CH}_{2} \mathrm{CH}\left(\mathrm{CH}_{3}\right)_{2}$ & $\mathrm{CH}_{2} \mathrm{CH}\left(\mathrm{CH}_{3}\right)_{2}$ & 19 \\
\hline
\end{tabular}


(a)

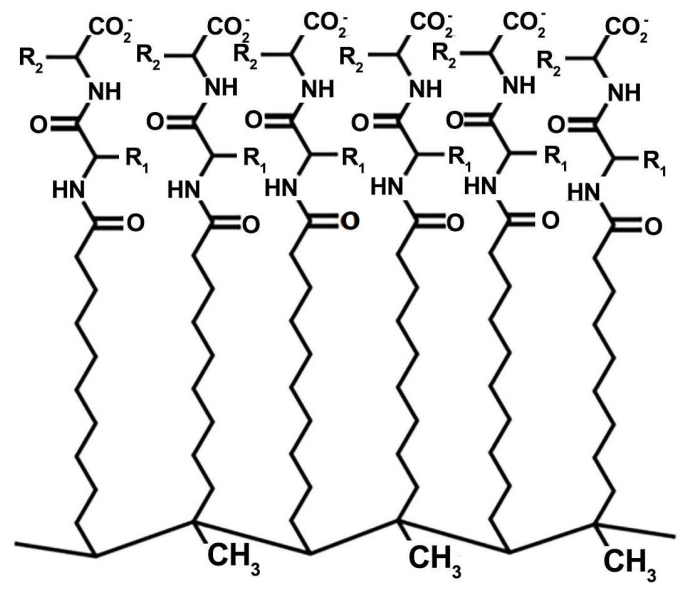

(b)



Figure 1. (a) Connectivity of surfactant monomers used to construct each AABMM, (b) Representative globular AABMM structure extracted from the SULL MD simulation.

predictions were compared to available experimental data. For example, distances between headgroup protons were compared to NMR NOESY experiments, the hydrodynamic radii of SULV and SUVL AABMM were compared to radii from NMR diffusion experiments, and CE enantiomer elution orders were compared to MD simulation free energies of solute binding. In all cases experimental and MD simulation results were found to be in good agreement. These comparisons, therefore, suggest that the MD simulation methods employed previously (and employed in this study as well) yield reasonable and accurate predictions of the AABMM structures.

AMBER 14 and the parm 99 force field were used to carry out $60.0 \mathrm{~ns}$ MD simulations for each AABMM. Each system contained the molecular micelle, sodium counterions and approximately 8000 TIP3P water molecules. The number of sodium ions was equal to the number of repeat units in each of the polymer systems [28]. Prior to the MD simulation production run, an energy minimization step was performed, followed by a 20 picosecond MD simulation to warm the system to $300 \mathrm{~K}$ and a one ns MD simulation to equilibrate to a pressure of one atmosphere. Each production run was $60.0 \mathrm{~ns}$ in duration, the time step was 
$2 \mathrm{fs}$, structures were stored every $0.2 \mathrm{ps}$, and cubic periodic boundary conditions were employed. Analyses of each MD simulation trajectory were done using the AMBER14 cpptraj utility. In all cases each timestep of the $60 \mathrm{~ns}$ MD simulation was included in the analysis.

In order to ensure that reasonable, globular molecular micelles were present throughout each MD simulation, representative structures were extracted by first calculating the root mean squared deviation (RMSD) of each MD simulation structure with respect to the average structure. Then representative structures having low RMSD values with respect to the average structure were extracted and examined at representative times from 0 to $60 \mathrm{~ns}$. This method was employed to choose specific structures from the MD simulation that were most similar to the average structure. All structures were then examined to confirm that the MD simulation produced reasonably appearing, globular AABMA structures. Again however, all of the solvent accessible surface area, dihedral angle, and distance measurements reported below resulted from analyses of each timestep of the $60 \mathrm{~ns}$ MD simulations. No quantitative results were calculated from the low RMDS structures.

\section{Results and Discussion}

As discussed above, the goal of this research is to characterize the factors responsible for the self-assembly, function, and higher ordered structure of AABMMs. These insights will then be used to build in silico predictive models that will identify the best AABMM selector for a given separation problem. Previous computational studies, however, have focused only on the AABMMs SULV, SUVL and SULL [21]-[27]. This study, therefore, seeks to expand that work by comparing the structures and properties of the nine different AABMM shown in Table 1. Figure 2(a) shows the backbone structure of the AABMM's investigated. Going forward, the amino acid attached to the hydrocarbon tail and containing the R1 side chain in Figure 2(a) will be referred to as either the "inside" amino acid or the "N-terminal" amino acid. The other amino acid containing the R2 side chain will be referred to as the "outside" or the "C-terminal" amino acid.

We first present analyses of the solvent accessible surface areas of the chiral centers in each dipeptide headgroup. These analyses were performed because stereoselective interactions between the AABMMs and chiral analyte enantiomers likely occur near the chiral centers. We, therefore, used MD simulations to probe the access that each $\mathrm{AABMM}$ provides to solvent molecules, and thus by extension to potential analyte enantiomers. Next, dihedral angle analyses were performed to characterize the conformations and conformational flexibilities of the AABMM dipeptide headgroups. Figures $2(\mathrm{~b})$-(d) define the headgroup dihedral angles $\omega 1, \omega 2$, and $\omega 3$ investigated here. These analyses were motivated in part by previous experimental work suggesting that steric hindrance may prevent two hydrophobic R-groups from orientating towards the hydrophobic 
<smiles>[R]C(NC([R])C([R])NC(=O)CCCCCCCCCCC)C(=O)O[NH3+]</smiles>

(b)<smiles>[R]C(NC(=O)CCCCCCCCCC)C(=O)NC([R])C(=O)O[NH3+]</smiles>

(c)<smiles>[R]C(NC(=O)CCCCCCCCCC)C([R])NC(=O)C(=O)O[NH3+]</smiles>

(d)

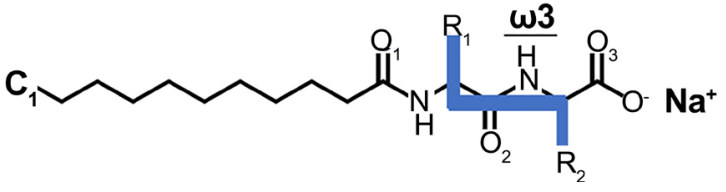

Figure 2. (a) Backbone structure of the amino acid-based molecular micelles, definition of the dihedral angles (b) $\omega 1$, (b) $\omega 2$, and (c) $\omega 3$.

micelle core to shield themselves from the aqueous environment [29] [30]. Finally, the distances between the headgroup alpha carbons and the micelle core (carbon atom $\mathrm{C} 1$ in Figure 2(a)) were monitored as a function of simulation time to study the headgroup structural flexibilities of the AABMMs under study.

\subsection{Solvent Accessible Surface Area Analyses}

SASA is defined as the surface area of an atom or molecule that is accessible or exposed to solvent molecules. SASA values are expected to be high if an atom or amino acid is in a polar or water exposed environment and low if an atom or amino acid is in a less polar environment shielded from solvent exposure. The SASA's of the AABMM alpha carbons were calculated and plotted versus simulation time. Figure 3 and Figure 4 show MD simulation-averaged SASA values for the nine AABMM's investigated. Results from these figures will now be discussed in order to demonstrate how the amino acid R-groups shield their respective alpha carbons from solvent molecules.

Figure 3(a) shows that when the amino acid in the second (C-terminal) position is held constant and the size of the R-group of the inside (N-terminal) amino acid is increased, the SASA's of the N-terminal alpha carbons decrease. For example, SASA values for SUAA, SUVA and SULA are 5.10, 4.32, and $2.84 \AA^{2}$, respectively. The same trends are observed for SUAV $\left(5.51 \AA^{2}\right)$, SUVV $\left(4.25 \AA^{2}\right)$ and SULV $\left(3.33 \AA^{2}\right)$ and SUAL $\left(5.01 \AA^{2}\right)$, SUVL $\left(4.24 \AA^{2}\right)$ and SULL $\left(2.39 \AA^{2}\right)$. Additionally, when the amino acid in the first ( $\mathrm{N}$-terminal) position is held constant and the sizes of R-groups on the outside (C-terminal) amino acids are increased the SASA of the C-terminal alpha carbons also decrease. These trends are shown in Figure 3(b). 

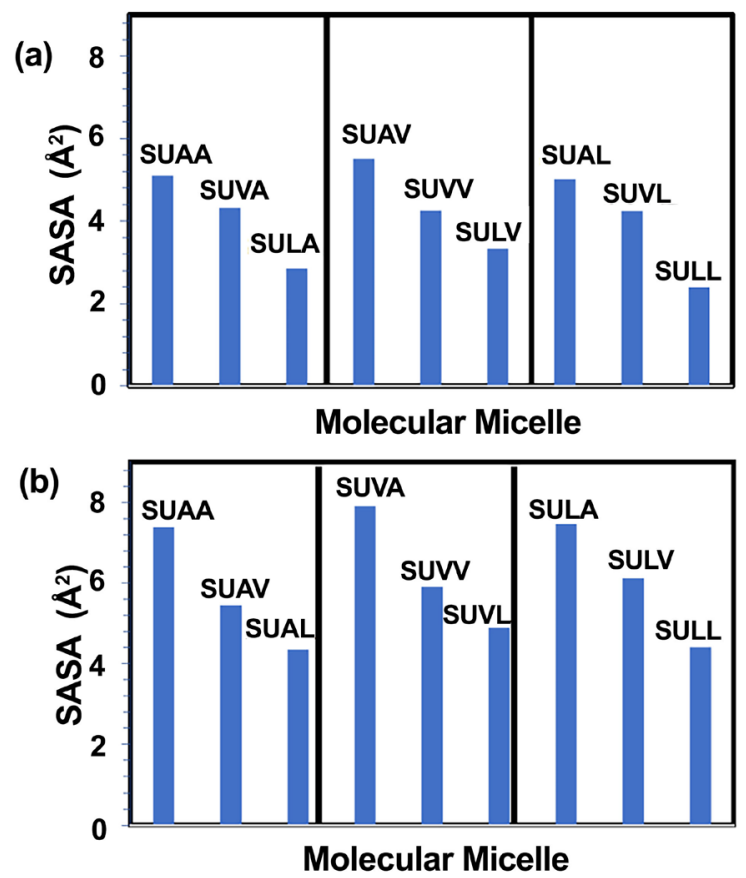

Figure 3. (a) Solvent accessible surface area plots showing the effect of increasing the size of $\mathrm{N}$-terminal R-Group on SASA of the N-terminal Ca atoms, (b) Solvent accessible surface area plots showing the effect of increasing the size of C-terminal R-Group on SASA of C-terminal Ca atoms.
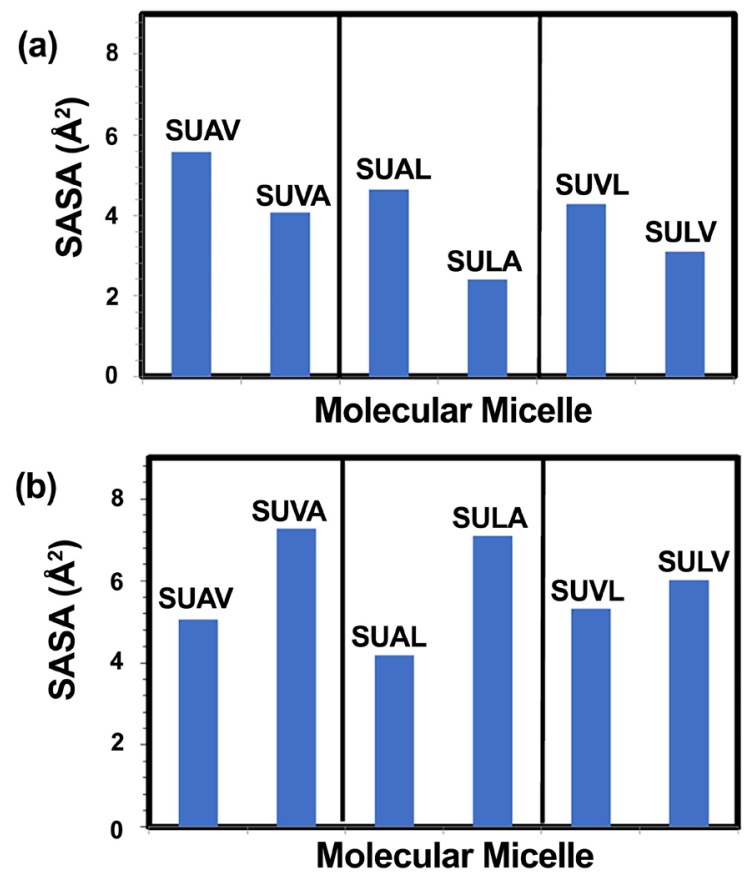

Figure 4. (a) Solvent accessible surface area plots showing the effect of amino acid order on SASA of the $\mathrm{N}$-terminal C $\alpha$ atoms, (b) Solvent accessible surface area plots showing the effect of amino acid order on SASA of C-terminal $\mathrm{C} \alpha$ atoms. 
The trends observed in Figure 3 are expected because SASA is a reflection of how shielded the alpha carbons are from solvent. This shielding could be due to the neighboring atoms (the larger the R-group the greater the shielding, theoretically) as well as, in our case, the location of the alpha carbon relative to the surface of the micelle. Note that in Figure 3(a) and Figure 3(b), the SASA values for the N-terminal alpha carbons tend to be smaller than the C-terminal alpha values. In fact, the average $\mathrm{N}$-terminal SASA values are $\sim 4.0 \pm 1.0 \AA^{2}$ while the averages for the C-terminal alpha carbons are $\sim 6.0 \pm 1.3 \AA^{2}$. Since the alpha carbons on the N-terminal amino acids are closer to each monomer's hydrophobic tail, they are also expected to be deeper inside the micellar core than the corresponding alpha carbons on the C-terminal amino acid.

Figure 4 illustrates the effect of headgroup amino acid order on alpha carbon SASA values. Figure 4(a) shows that the SASA's of the N-terminal alpha carbon are always less when the larger of the two amino acids is in the first or $\mathrm{N}$-terminal position. In contrast, the SASA of the C-terminal alpha carbon is always less when the smaller of the two amino acids is in the N-terminal position (see Figure $4(\mathrm{~b})$ ). For the same reasons as discussed above, these trends are as expected.

\subsection{Dihedral Angles Analyses}

Results from MD simulation dihedral angle analyses will now be presented in order to more fully describe the conformations and structural features of the AABMMs under study. The dihedral angles investigated are defined in Figure 2. The first angle $\omega 1$ (Figure 2(b)) is the dihedral angle between the first atom on the $\mathrm{R} 1$ side chain, the $\mathrm{N}$-terminal alpha carbon, the $\mathrm{N}$-terminal carbonyl carbon, and the carbonyl oxygen (O2). The second dihedral angle, $\omega 2$, (Figure 2(c)) is between atom $\mathrm{O} 2$, the $\mathrm{N}$-terminal carbonyl carbon, the $\mathrm{C}$-terminal alpha carbon, and the first atom of the C-terminal side chain. Finally, $\omega 3$ is the dihedral angle formed by the first atom of the $\mathrm{N}$-terminal side chain, the $\mathrm{N}$-terminal alpha carbon, the C-terminal alpha carbon, and the first atom of the C-terminal side chain. The later angle was chosen to probe the relative orientations of the two $\mathrm{R}$ groups in each dipeptide headgroup.

Figure 5 shows $\omega 1$ and $\omega 2$ dihedral angle histograms for the AABMM's SUAA and SULL. Analogous histograms for the seven other AABMM are shown in Supplemental Figure 1. The data in each of these histogram plots was also used to calculate the average dihedral angle values reported in Table 2. Each Figure 5 and Supplemental Figure 1 histogram was generated by first calculating values for the dihedral angles $\omega 1, \omega 2$, and $\omega 3$ for each time step of the MD simulations. Histograms of these data were then prepared to show the preferred dihedral angle values and to investigate the variability of each dihedral angle during the MD simulations. The maxima of each peak in the histograms were then determined along with the relative peak areas for histograms showing bimodal behavior. These values are presented in Table 2. Overall, Figure 5 (and Supplemental Figure 1) and Table 2 show that the dihedral angle $\omega 1$ took on 

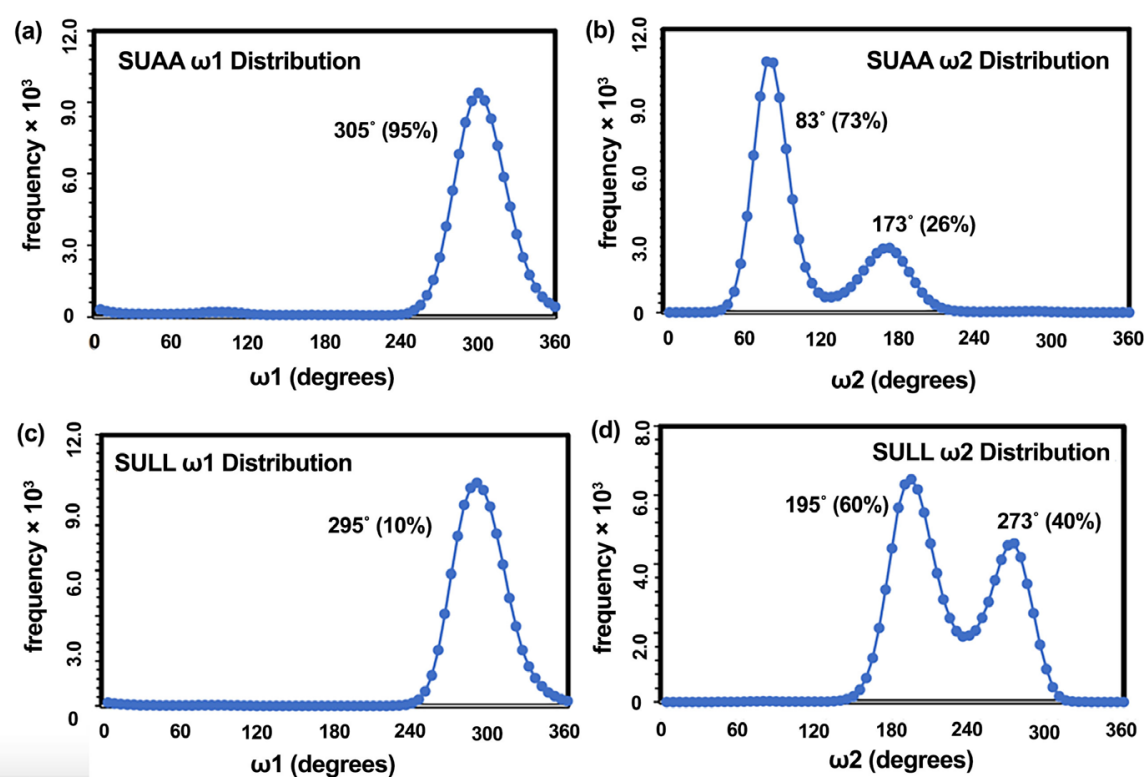

Figure 5. Dihedral angle histograms for (a) $\omega 1$ in SUAA, (b) $\omega 2$ in SUAA, (c) $\omega 1$ in SULL, and (d) $\omega 2$ SULL.

Table 2. Average dihedral angles and relative major and minor peak areas from the AABMM dihedral angle distributions.

\begin{tabular}{cccccccccccc}
\hline & \multicolumn{1}{c}{$\boldsymbol{\omega 1}$ (degrees) } & \multicolumn{3}{c}{$\boldsymbol{\omega}$ 2 (degrees) } & \multicolumn{3}{c}{$\boldsymbol{\omega 3}$ (degrees) } \\
\cline { 2 - 11 } Surfactant & $\begin{array}{c}\text { Major } \\
\text { Peak }\end{array}$ & $\%$ & $\begin{array}{c}\text { Major } \\
\text { Peak }\end{array}$ & $\%$ & $\begin{array}{c}\text { Minor } \\
\text { Peak }\end{array}$ & $\%$ & $\begin{array}{c}\text { Major } \\
\text { Peak }\end{array}$ & $\%$ & $\begin{array}{c}\text { Minor } \\
\text { Peak }\end{array}$ & $\%$ \\
\hline SUAA & 305 & 95 & 85.5 & 73 & 172.5 & 26 & 15 & 72 & 107.5 & 27 \\
SUAV & 295 & 98 & 87.5 & 74 & 177.5 & 26 & 15 & 60 & 125 & 40 \\
SUAL & 305 & 99 & 95 & 58 & 175 & 41 & 20 & 59 & 115 & 41 \\
SUVA & 295 & 100 & 85 & 82 & 172.5 & 18 & 12.5 & 83 & 90 & 17 \\
SUVV & 290 & 99 & 85 & 87 & 172.5 & 13 & 10 & 87 & 115 & 13 \\
SUVL & 297.5 & 100 & 90 & 70 & 165 & 30 & 15 & 64 & 85 & 36 \\
SULA & 300 & 99 & 82.5 & 79 & 170 & 20 & 17.5 & 78 & 95 & 22 \\
SULV & 295 & 100 & 90 & 77 & 170 & 23 & 12.5 & 78 & 110 & 22 \\
SULL & 295 & 100 & 195 & 30 & 272.5 & 40 & 112.5 & 59 & 195 & 40
\end{tabular}

*average and standard deviations of averages in highlighted boxes excluded SULL data.

very similar values for all AABMM's investigated. In fact, averaging the $\omega 1$ value in Table 2 for all nine AABMM's yielded $298^{\circ} \pm 5^{\circ}$. The stability and relatively narrow distribution of the $\omega 1$ dihedral angles is likely because the atoms forming $\omega 1$ are close to the micellar core and, therefore, in a sterically restricted environment.

In contrast, Figure 5 and Supplemental Figure 1 show that each $\omega 2$ dihedral angle distribution displayed bimodal behavior. It is also interesting to note that all of the AABMM systems examined, except SULL, also had very similar aver- 
age $\omega 2$ values. For example, the average $\omega 2$ dihedral angles from the larger or major histogram peaks, with the exception of SULL, were $88^{\circ} \pm 4^{\circ}$. The dihedral angles corresponding to the smaller or minor histogram peaks were $172^{\circ} \pm 4^{\circ}$. Again averages were calculated for all AABMM's except SULL.

With SULL, however, the major $\omega 2$ peak in the dihedral angle distribution was centered at $195^{\circ}$ with a smaller, minor peak at $272.5^{\circ}$. These SULL $\omega 2$ dihedral angle values were, therefore, approximately $100^{\circ}$ larger than the corresponding dihedrals for the other eight AABMM's. This significant difference is likely attributable to steric hindrance between the two relatively bulky leucine moieties. It should be noted, however, that while SULL had significantly larger $\omega 2$ values than the other AABMM's, the difference between the SULL major and minor dihedral angle peaks was $\sim 91^{\circ}$ while the corresponding average difference for the other AABMM's in this study was $\sim 84^{\circ}$. Thus, while there is a relatively large shift in $\omega 2$ values for SULL compared to the other systems, the differences between the major and minor peaks of the various $\omega 2$ angle distributions are approximately the same. Analyses of $\omega 3$ dihedral angles showed similar behavior.

While $\omega 1$ and $\omega 2$ represent the dihedral angles formed by only one of the amino acid R-groups and other headgroup atoms, $\omega 3$ represents the dihedral angle between both R-groups and the dipeptide backbone. The $\omega 3$ dihedral angle distributions for SUAA and SULL are shown in Figure 6. Corresponding histograms for the other seven AABMM's are shown in Supplemental Figure 2. Note that as was observed for $\omega 1$ and $\omega 2$, the $\omega 3$ histograms show peaks at very similar values for all AABMM except SULL. The average major $\omega 3$ dihedral angle for the AABMM's (excluding SULL) was $15^{\circ} \pm 3^{\circ}$, while the minor peak was centered at $105^{\circ} \pm 14^{\circ}$. Figure 7 (a) and Figure 7 (b) show two orientations of an AABMM monomer with an $\omega 3$ dihedral angle equal to $15^{\circ}$. This relatively small dihedral angle suggests that when Alanine or Valine is present in the dipeptide headgroup there are relatively few steric interactions between the two $\mathrm{R}$ groups and thus the headgroup adopts a relatively small $\omega 3$ dihedral angle.

As with $\omega 1$ and $\omega 2$, a significant shift in $\omega 3$ was observed for SULL compared to the other AABMMs. The dihedral angle associated with the major and minor peaks in the $\omega 3$ SULL dihedral angle distributions were $112.5^{\circ}$ and $195^{\circ}$, respectively. The larger SULL major dihedral $\left(112.5^{\circ}\right.$ versus $\left.15^{\circ}\right)$ suggests that the Leucine side chains do interact sterically when they occupy the R1 and R2 headgroup positions. These interactions in turn cause the headgroup to adopt a conformation that places the two $\mathrm{R}$ groups farther from one another when compared to the other eight AABMM in this study.

Finally, the $\omega 3$ dihedral angle distributions were similar to the distributions observed for $\omega 1$ and $\omega 2$ in that the differences between the major and minor peaks were similar for all AABMM's investigated. For example, the difference between the average major and minor $\omega 3$ peaks, $15^{\circ}$ and $105^{\circ}$ respectively, for all of surfactants excluding SULL was $\sim 90^{\circ}$, while the difference between the major 

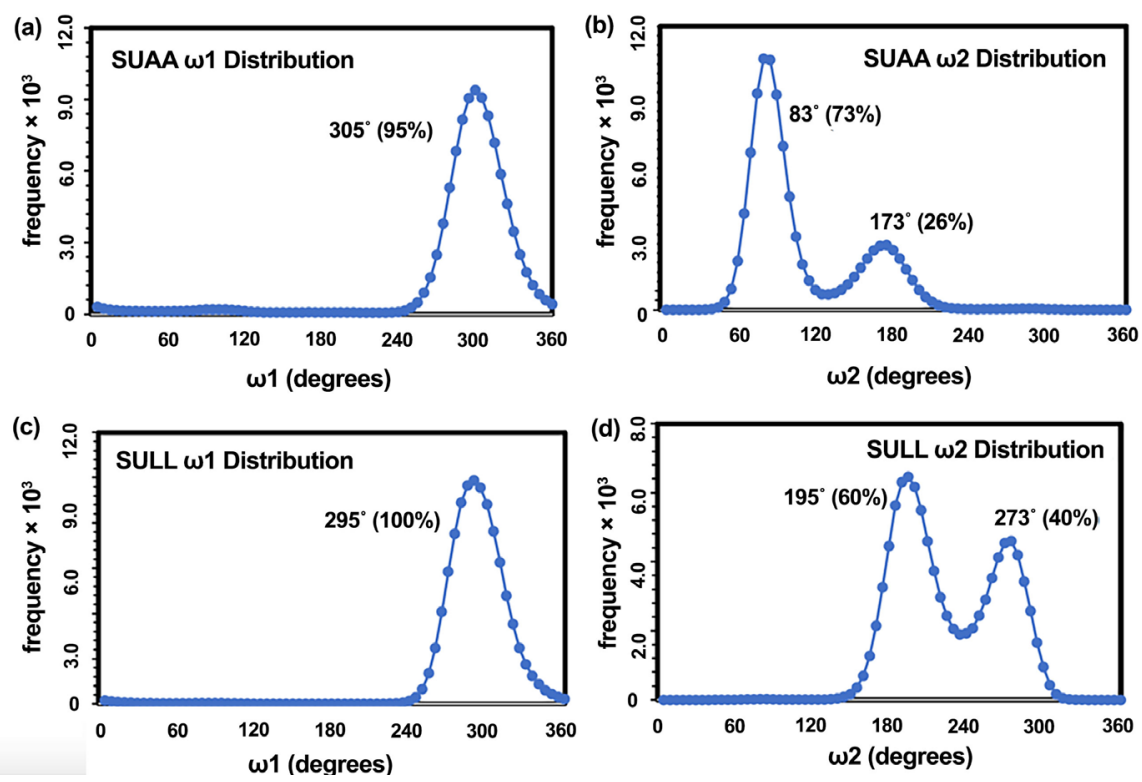

Figure 6. Dihedral angle histograms for (a) $\omega 3$ in SUAA and (b) $\omega 3$ in SULL.
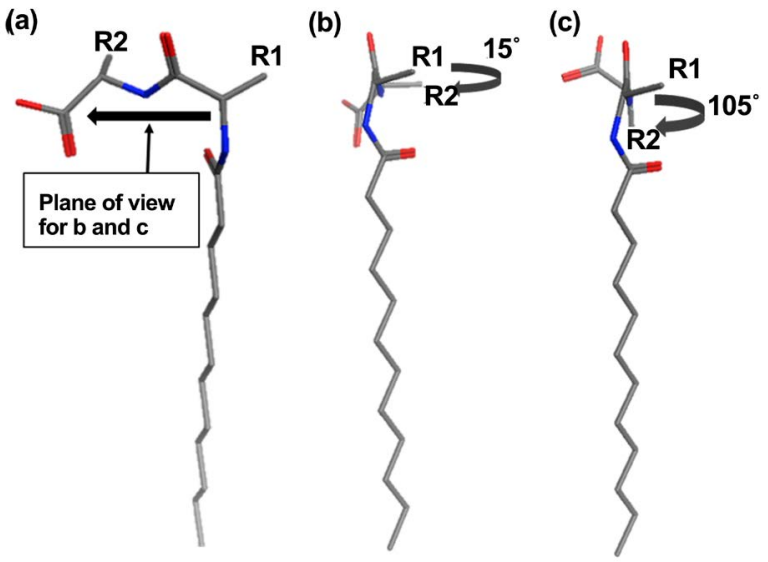

Figure 7. (a) AABMM monomer structure showing the relative orientation of side chains R1 and R2, (b) AABMM monomer structure with an $\omega 3$ dihedral angle of $15^{\circ}$, (c) AABMM structure with an $\omega 3$ dihedral angle of $105^{\circ}$.

$\omega 3$ peak $\left(112.5^{\circ}\right)$ and minor $\omega 3$ peak $\left(195^{\circ}\right)$ for SULL was $\sim 82.5^{\circ}$. Thus, as with dihedrals $\omega 1$ and $\omega 2$, the difference between the major and minor $\omega 3$ peaks for SULL and the other surfactants were approximately the same.

\subsection{Conformational Flexibility}

To examine the headgroup structural flexibilities of the AABMMs under study, the distances between the alpha carbons on the N-terminal and C-terminal amino acids and the first carbon on each surfactant monomers' hydrocarbon chain (atom C1 in Figure 2(a)) were measured as a function of MD simulation time. Table 3 shows average and standard deviation values for these distances. Note that two distances are reported for each AABMM: one from $\mathrm{C} 1$ to the 
Table 3. Averages and standard deviations for $\mathrm{C} 1$ to $\mathrm{N}$-terminal $\mathrm{Ca}$ distances and $\mathrm{C} 1$ to C-terminal Ca distances.

\begin{tabular}{|c|c|c|c|c|}
\hline & $\begin{array}{c}\text { Average Distance } \\
\text { between } \mathrm{C} 1 \text { and } \\
\text { N-Terminal Ca }(\AA)\end{array}$ & $\begin{array}{c}\text { Standard Deviation of } \\
\text { C1 to N-terminal Ca } \\
\text { Distance }(\AA)\end{array}$ & $\begin{array}{c}\text { Average Distance } \\
\text { between C1 and } \\
\text { C-Terminal Ca }(\AA)\end{array}$ & $\begin{array}{c}\text { Standard Deviation of } \\
\text { C1 to C-terminal Ca } \\
\text { Distance }(\AA)\end{array}$ \\
\hline SUAA & 11.54 & 1.81 & 13.18 & 2.27 \\
\hline AUAV & 10.93 & 1.69 & 11.77 & 2.72 \\
\hline SUAL & 11.02 & 0.44 & 12.04 & 2.21 \\
\hline SUVA & 9.53 & 0.70 & 12.02 & 0.58 \\
\hline SUVV & 9.56 & 0.60 & 12.04 & 0.56 \\
\hline SUVL & 9.16 & 0.66 & 11.21 & 0.56 \\
\hline SULA & 10.46 & 0.49 & 13.75 & 0.45 \\
\hline SULV & 9.94 & 0.64 & 12.31 & 0.51 \\
\hline SULL & 11.55 & 0.35 & 13.63 & 0.47 \\
\hline
\end{tabular}

N-terminal $\mathrm{Ca}$ and the other to the $\mathrm{C}$ terminal $\mathrm{Ca}$. It should also be noted that the average distances in Table 3 were averaged over both the timesteps of the $60.0 \mathrm{~ns} \mathrm{MD}$ simulation and each surfactant monomer making up the respective AABMM's. Standard deviations were calculated in a similar manner. Table 3 shows that the N-terminal $\mathrm{C} \alpha$ to $\mathrm{C} 1$ distances fell in the range of 9.16 to $11.55 \AA$, while the C-terminal $\mathrm{C} a$ to $\mathrm{C} 1$ distances ranged from 11.21 - $13.75 \AA$.

While most of the AABMM's had similar $\mathrm{Ca}$ to $\mathrm{C} 1$ distances, the Table 3 standard deviations values showed greater variability. Furthermore, these standard deviations would be expected to be governed in part by the conformational or structural flexibilities of the AABMM headgroups. For example, if a headgroup adopted a relatively rigid conformation that changed little during the MD simulation, the distances between the alpha carbons and $\mathrm{C} 1$ would be expected to remain relatively constant and the corresponding Table 3 standard deviations would be small. If the headgroup instead showed more flexible or dynamic behavior, there would be more variability in the $\mathrm{C} \alpha$ to $\mathrm{C} 1$ distances leading to larger Table 3 standard deviation values.

Figure $8(\mathrm{a})$ plots the standard deviations of the distances between the $\mathrm{N}$-terminal $\mathrm{Ca}$ and $\mathrm{C} 1$ for each AABMM. Note that, with the exception SUAA and SUAV, the standard deviation values are all relatively small and constant. These results indicate that the N-terminal $\mathrm{C} a$ to $\mathrm{C} 1$ distances showed little variability and the orientation of the R1 side chains likely remain relative constant during the MD simulations. SUAA and SUAV with smaller amino acid side chains, in contrast, have much larger standard deviations values indicating that these headgroups are more conformationally flexible, leading to the greater variability observed in the $\mathrm{N}$-terminal $\mathrm{Ca}$ to $\mathrm{C} 1$ distances. SUAL, however, has a much smaller corresponding standard deviation than either SUAA or SUAV. This behavior is likely due to the fact that the SUAL leucine R-group, which is fairly hydrophobic, is facing the hydrophobic core of the micelle. The resulting 
(a)

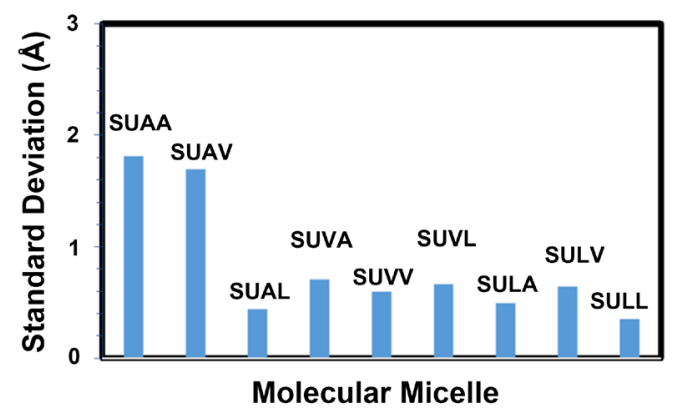

(b)

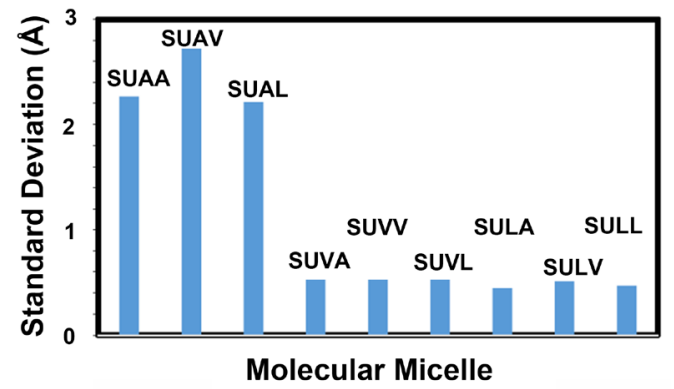

Figure 8. (a) Standard deviations of the distances between the N-terminal Ca and C1, (b) Standard deviations of the distances between the C-terminal Ca and C1.

steric hindrance, therefore, restricts the motion of the $\mathrm{N}$-terminal amino acid leading to a relatively stable $\mathrm{N}$-terminal $\mathrm{C} \alpha$ to $\mathrm{C} 1$ distance.

Finally, Figure 8(b) plots the standard deviations of the C-terminal $\mathrm{Ca}$ to $\mathrm{C} 1$ distances for each AABMM. Figure 8(b) results show that when alanine is in the $\mathrm{N}$-terminal position (as in SUAA, SUAV, and SUAL) the C-terminal amino acid has more freedom of motion than if the N-terminal amino acid is Valine or Leucine. It is also interesting to note that when comparing Figure 8(a) to Figure 8(b), the N-terminal amino acids generally have less freedom of motion compared to the C-terminal amino acids. This result would be expected since the R1 side chains are closer to the micellar core and thus in the much more sterically restricted environment.

\section{Conclusions}

MD simulations were used to investigate the structural features of nine different AABMMs. The study found that in AABMM where the C-terminal amino acid remained constant and the size of the $\mathrm{N}$-terminal amino acid's R-group was increased, the SASAs of the N-terminal alpha carbon decreased. Similarly, when the N-terminal amino acid remained constant and the size of the C-terminal amino acid's R-group increased, the SASAs of the C-terminal alpha carbon also decreased. This behavior models expected trends in that an increase in the size of a headgroup amino acid's R-group would be expected to restrict solvent access to that amino acid's alpha carbon. The study also found that the SULL headgroup adopted a significantly different conformation than the other AABMM investigated. Differences were quantified by measuring the dihedral 
angles $\omega 1, \omega 2$, and $\omega 3$ shown in Figure 2. This behavior is likely due to increased steric hindrance experienced by the bulky leucine moieties. Finally, the conformational or structural flexibilities of the AABMM headgroups were also examined. It was found that when alanine is in the $\mathrm{N}$-terminal position, the headgroups' C-terminal amino acids have greater flexibility. In addition, for all AABMM's investigated, the headgroup N-terminal amino acids had less freedom of motion than the C-terminal amino acids. This behavior is expected as the $\mathrm{N}$-terminal environment is closer to the micellar core.

Overall, the MD simulations reported here for nine different AABMM produced reasonable, consistent, and expected trends in both their structural and dynamic properties. Therefore, the structures in this study will be used, along with additional computational tools, to begin building a database of QSERR and other in silico tools that will evaluate and compare the effectiveness of different AABMM's in chiral capillary electrophoretic separations.

\section{Acknowledgements}

This work was supported by grant from National Institute of Health (No. 2U54MD007597) and grants from National Science Foundation under grant numbers: 1708959, 1709394, and 1709680. The research was also supported by Office of Naval Research under Grants N00014-18-1-2145 and N00014-19-1-2602, and a Robert A. Welch Chemistry Departmental Grant awarded to Texas A\&M University Corpus Christi Chemistry Program. In addition, Dr. Kevin Morris acknowledges the generosity of the Ralph E. Klingenmeyer family.

\section{Conflicts of Interest}

The authors declare no conflicts of interest regarding the publication of this paper.

\section{References}

[1] Gumustas, M., Ozkan, S.A. and Chankyetadze, B. (2018) Analytical and Preparative Scale Separation of Enantiomers of Chiral Drugs by Chromatography and Related Methods. Current Medicinal Chemistry, 25, 4152-4188. https://doi.org/10.2174/0929867325666180129094955

[2] Gus'kov, V.Y. and Maistrenko, V.N. (2018) New Chiral Stationary Phases: Preparation, Properties, and Applications in Gas Chromatography. Analytical Chemistry, 73, 937-945. https://doi.org/10.1134/S1061934818100027

[3] Ding, X., Lin, S., Weng, H. and Liang, J. (2018) Separation and Determination of the Enantiomers of Lactic Acid and 2-Hydroxyglutaric Acid by Chiral Derivatization Combined with Gas Chromatography and Mass Spectrometry. Journal of Separation Science, 41, 2576-2584. https://doi.org/10.1002/jssc.201701555

[4] Zenkevich, I.G. and Nosova, V.E. (2018) Characterization of Dialkyl Phosphites by Gas Chromatography-Mass Spectrometry. Analytical Chemistry, 73, 1162-1176. https://doi.org/10.1134/S1061934818090150

[5] Lin, M., Zhao, H., Zhang, Z., Li, Z., Mao, L., Zhang, R. and Wang, Y. (2019) Deriva- 
tization Reagent-Assisted Enantioseparation of 3-Hydroxyaspartate with Two Chiral Centers in Rat Cerebrospinal Fluid by Capillary Electrophoresis-Mass Spectrometry. Analytical Chimicam Acta, 1047, 257-266.

https://doi.org/10.1016/j.aca.2018.09.070

[6] Kulsing, C., Nolvachai, Y., Wong, Y.F., Glouzman, M.I. and Marriott, P.J. (2018) Observation and Explanation of Two-Dimensional Interconversion of Oximes with Multiple Heart-Cutting Using Comprehensive Multidimensional Gas Chromatography. Journal of Chromatography A, 1546, 97-105.

https://doi.org/10.1016/j.chroma.2018.02.035

[7] Mimura, K., Okada, R. and Nishida, T. (2018) Chiral Gas Chromatography of 2, 5-Diketopiperazines Following a Ring-Opening Derivatization Method for Complete Isomeric Separation. Journal of Chromatography $A, 1566,118-123$.

https://doi.org/10.1016/j.chroma.2018.06.051

[8] Teixeira, J., Tiritan, M.E., Pinto, M.M.M. and Fernandes, C. (2019) Chiral Stationary Phases for Liquid Chromatography: Recent Developments. Molecules, 24, 865. https://doi.org/10.3390/molecules24050865

[9] Khundadze, N., Pantsulaia, S., Fanali, C., Farkas, T. and Chankvetadze, B. (2018) On Our Way to Sub-Second Separations of Enantiomers in High-Performance Liquid Chromatography. Journal of Chromatography A, 1572, 37-43. https://doi.org/10.1016/j.chroma.2018.08.027

[10] Zhang, Y., Du, Y., Yu, T., Feng, Z. and Chen, J. (2019) Investigation of Dextrin-Based Synergistic System with Chiral Ionic Liquids as Additives for Enantiomeric Separation in Capillary Electrophoresis. Journal of Pharmaceutical and Biomedical Analysis, 164, 413-420. https://doi.org/10.1016/j.jpba.2018.10.002

[11] Shapovalova, E.N., Fedorova, I.A., Anan'eva, I.A. and Shpigun, O.A. (2018) Macrocyclic Antibiotics as Chiral Selectors in High-Performance Liquid Chromatography and Capillary Electrophoresis. Journal of Analytical Chemistry, 73, 1064-1075. https://doi.org/10.1134/S1061934818110114

[12] Hu, Y. and Wei, X. (2018) Enantioseparation and Determination of Norepinephrine, Epinephrine and Isoproterenol by Capillary Electrophoresis-indirect Electrochemiluminescence in Human Serum. Current Analytical Chemistry, 14, 504-511. https://doi.org/10.2174/1573411013666170929150919

[13] Ramos, Z., Rothbauer, G.A., Turner, J., Corbin Lewis, C., Morris, K., Billiot, E., Billiot, F. and Fang, Y. (2018) Comparison of Chiral Recognition of Binaphthyl Derivatives with L-Undecyl-Leucine Surfactants in the Presence of Arginine and Sodium Counterions. Journal of Chromatographic Sciences, 57, 54-62.

https://doi.org/10.1093/chromsci/bmy080

[14] Shamsi, S.A., Valle, B.C., Billiot, F. and Warner, I.M. (2003) Polysodium N-Undecanoyl-L-Leucylvalinate: A Versatile Chiral Selector for Micellar Electrokinetic Chromatography. Analytical Chemistry, 75, 379-387. https://doi.org/10.1021/ac020386r

[15] Billiot, E., Billiot, F.H. and Warner, I.M. (2008) Optimization of 12 Chiral Analytes with 8 Polymeric Surfactants. Journal Chromatographic Science, 46, 757-763. https://doi.org/10.1093/chromsci/46.9.757

[16] Billiot, F.H. Billiot, E., Ng, Y. and Warner, I.M. (2006) Chiral Separation of Norlaudanosoline, Laudanosoline, Laudanisone, Chlorothalidone, and Three Benzoin Derivatives Using Amino Acid Based Molecular Micelles. Journal of Chromatographic Science, 44, 64-69. https://doi.org/10.1093/chromsci/44.2.64

[17] Yarabe, Y., Billiot, E.J. and Warner, I.M. (2000) Enantiomeric Separations by Use of 
Polymeric Surfactant Electrokinetic Chromatography. Journal of Chromatography $A$, 875, 179-206. https://doi.org/10.1016/S0021-9673(00)00064-9

[18] Billiot, F.H., Billiot, E.J. and Warner. I.M. (2001) Comparison of Monomeric and Polymeric Amino Acid Based Surfactants for Chiral Separations. Journal of Chromatography $A$, 922, 329-338. https://doi.org/10.1016/S0021-9673(01)00865-2

[19] Billiot, F.H., McCarroll, M.C., Billiot, E.J. and Warner, I.M. (2004) Chiral Recognition of Binaphthyl Derivatives Using Electrokinetic Chromatography and Steady-State Fluorescence Anisotropy: Effect of Temperature. Electrophoresis, 25, 753-757. https://doi.org/10.1002/elps.200305649

[20] Yu, R.B. and Quirino, J.P. (2019) Chiral Selectors in Capillary Electrophoresis: Trends during 2017-2018. Molecules, 24, 1135-1153. https://doi.org/10.3390/molecules24061135

[21] Morris, K.F., Billiot, F.H., Billiot, E.J., Lipkowitz, K., Southerland, W. and Fang, Y. (2012) Investigation of Chiral Molecular Micelles by NMR Spectroscopy and Molecular Dynamics Simulation. Open Journal of Physical Chemistry, 2, 240-251. https://doi.org/10.4236/ojpc.2012.24032

[22] Morris, K.F., Billiot, F.H., Billiot, E.J., Lipkowitz, K., Southerland, W. and Fang, Y. (2013) A Molecular Dynamics Simulation Study of Two Dipeptide Based Molecular Micelles: Effect of Amino Acid Order. Open Journal of Physical Chemistry, 3, 20-29. https://doi.org/10.4236/ojpc.2013.31004

[23] Morris, K.F., Billiot, E.J., Billiot, F.H., Garcia, A., Lipkowitz, K.B., Southerland, W.M. and Fang, Y. (2014) A Molecular Dynamics Simulation Study of the Association of 1,1'-Binaphthyl-2,2'-Diyl Hydrogen Phosphate Enantiomers with a Chiral Molecular Micelle. Chemical Physics, 439, 36-43.

https://doi.org/10.1016/j.chemphys.2014.05.004

[24] Morris, K.F., Billiot, E.J., Billiot, F.H., Lipkowitz, K.B., Southerland, W.M. and Fang, Y. (2015) Molecular Dynamics Simulation and NMR Investigation of the Association of the $\beta$-Blockers Atenolol and Propranolol with a Chiral Molecular Micelle. Chemical Physics, 457, 133-146. https://doi.org/10.1016/j.chemphys.2015.05.024

[25] Lewis, C., Hughes, B.H., Vasquez, M., Wall, A.M., Northrup, V.L., Witzleb, T.J., Billiot, E.J., Fang, Y., Billiot, F.H. and Morris, K.F. (2016) Effect of $\mathrm{pH}$ on the Binding of Sodium, Lysine, and Arginine Counterions to L-Undecyl Leucinate Micelles. Journal of Surfactants and Detergents, 19, 1175-1188. https://doi.org/10.1007/s11743-016-1875-y

[26] Morris, K.F., Billiot, E.J., Billiot, F.H., Ingle, J.A., Zack, S.R., Krause, K.B., Lipkowitz, K.B., Southerland, W.M. and Fang, Y. (2018) Investigation of Chiral Recognition by Molecular Micelles with Molecular Dynamics Simulations. Journal of Dispersion Science and Technology, 39, 45-54. https://doi.org/10.1080/01932691.2017.1292462

[27] Morris, K.F., Billiot, E.J., Billiot, F.H., Ingle, J.A., Krause, K.B., Lewis, C.R., Lipkowitz, K.B., Southerland, W.M. and Fang, Y. (2019) Using Molecular Dynamics Simulations to Identify the Key Factors Responsible for Chiral Recognition by an Amino Acid-Based Molecular Micelle. Journal of Dispersion Science and Technology, 40, 716-727. https://doi.org/10.1080/01932691.2018.1479267

[28] Amber Case, D.A., Babin, V., Berryman, J.T., Betz, R.M., Cai, Q., Cerutti, D.S., Cheatham, T.E., Darden, T.A., Duke, R.E., et al. (2014) AMBER 14. University of California, San Francisco, CA.

[29] Billiot, F., McCarroll, M.E., Billiot, E.J., Rugutt, J.K., Morrism, K.F. and Warner, I.M. (2002) Comparison of the Aggregation Behavior of Fifteen Polymeric and 
Monomeric Dipeptide Surfactants in Aqueous Solution. Langmuir, 18, 2993-2997. https://doi.org/10.1021/la0110592

[30] Thibodeaux, S.J., Billiot, E.J. and Warner, I.M. (2002) Enantiomeric Separations Using Poly (L-Valine) and Poly (L-Leucine) Surfactants: Investigation of Steric Factors Near the Chiral Center. Journal of Chromatography A, 966, 179-186. https://doi.org/10.1016/S0021-9673(02)00747-1 

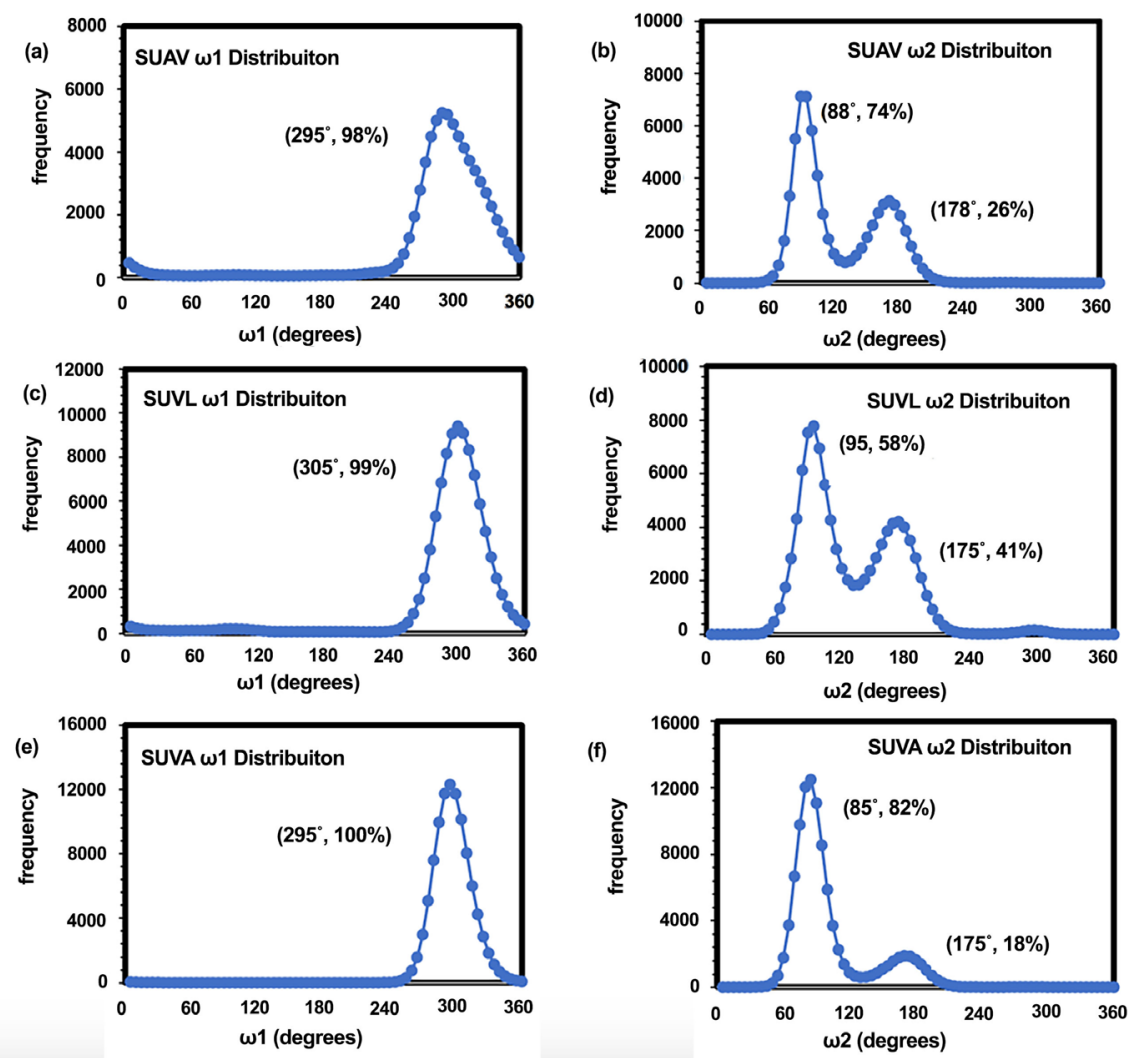

(Part 1)
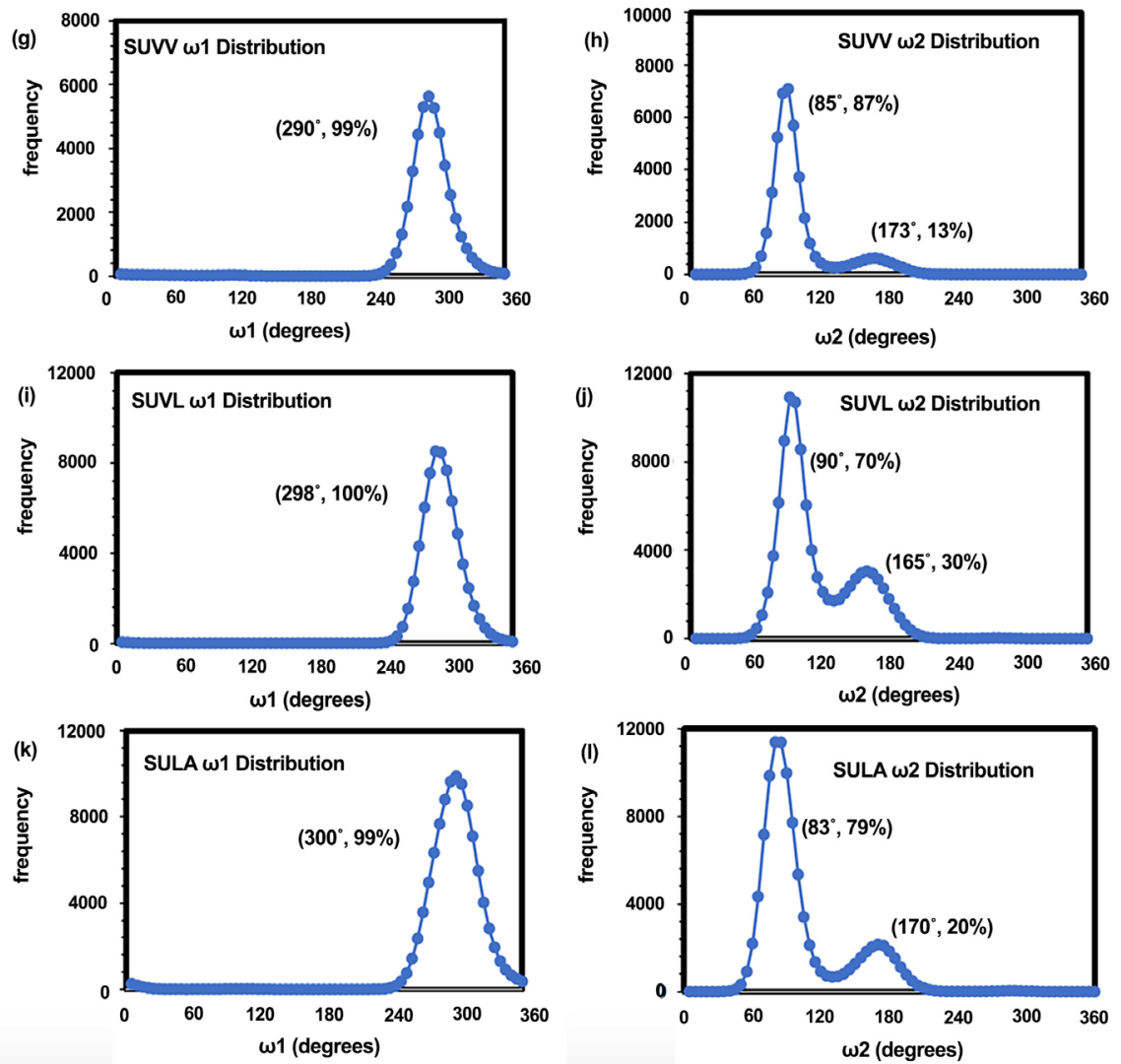

(Part 2) 

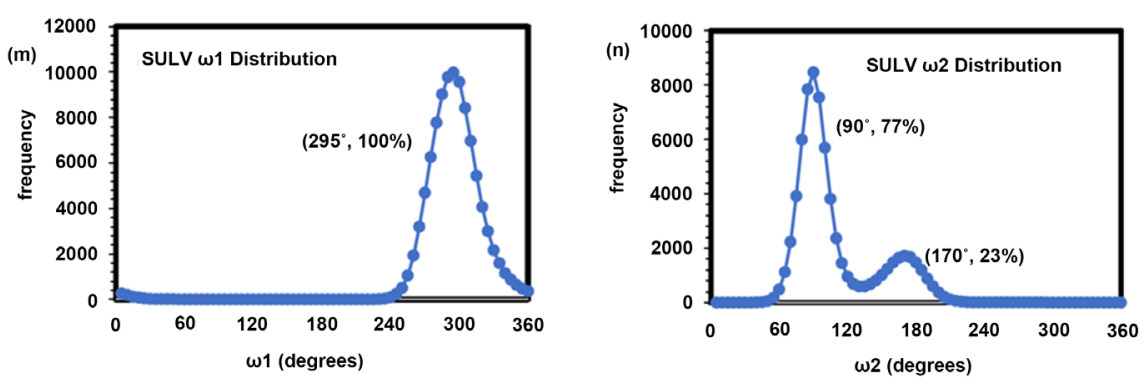

(Part 3)

Supplemental Figure 1. (Part 1) $\omega 1$ and $\omega 2$ dihedral angle distribution histograms for (a)/(b) SUAV, (c)/(d) SUAL, (e)/(f) SUVA. (Part 2) $\omega 1$ and $\omega 2$ dihedral angle distribution histograms for (g)/(h) SUVV, (i)/(j) SUVL, (k)/(l) SULA. (Part 3) $\omega 1$ and $\omega 2$ dihedral angle distribution histograms for $(\mathrm{m}) /(\mathrm{n}) \mathrm{SULV}$. 

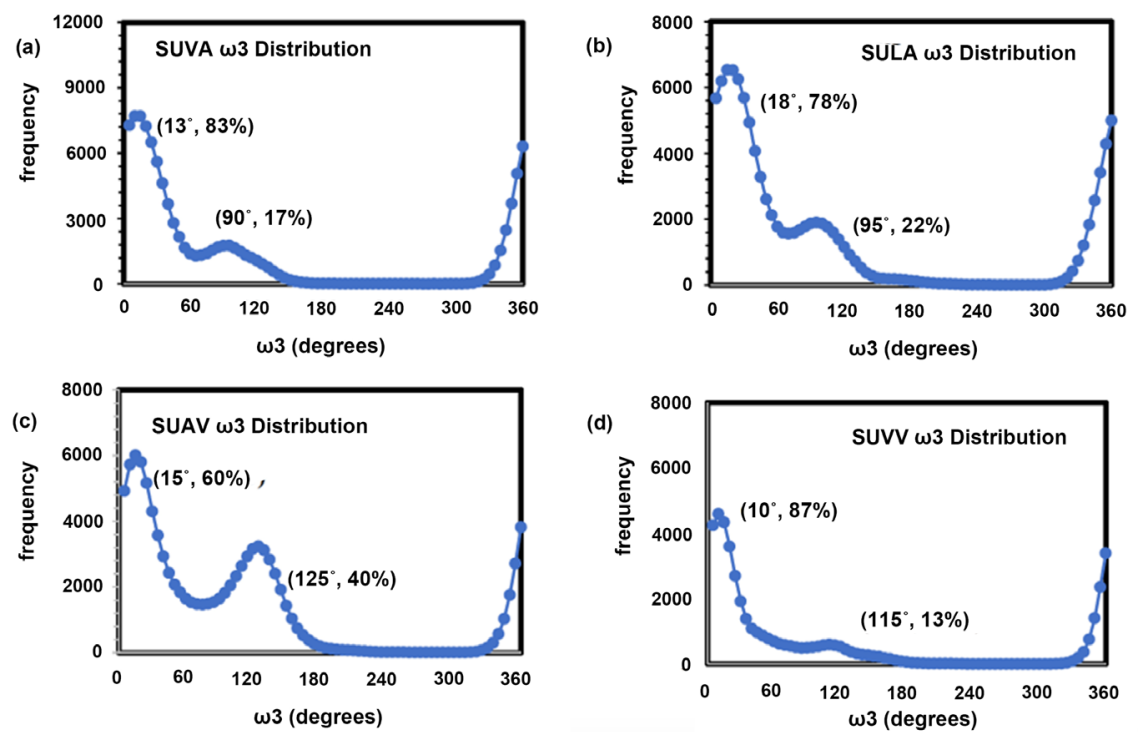

(Part 1)
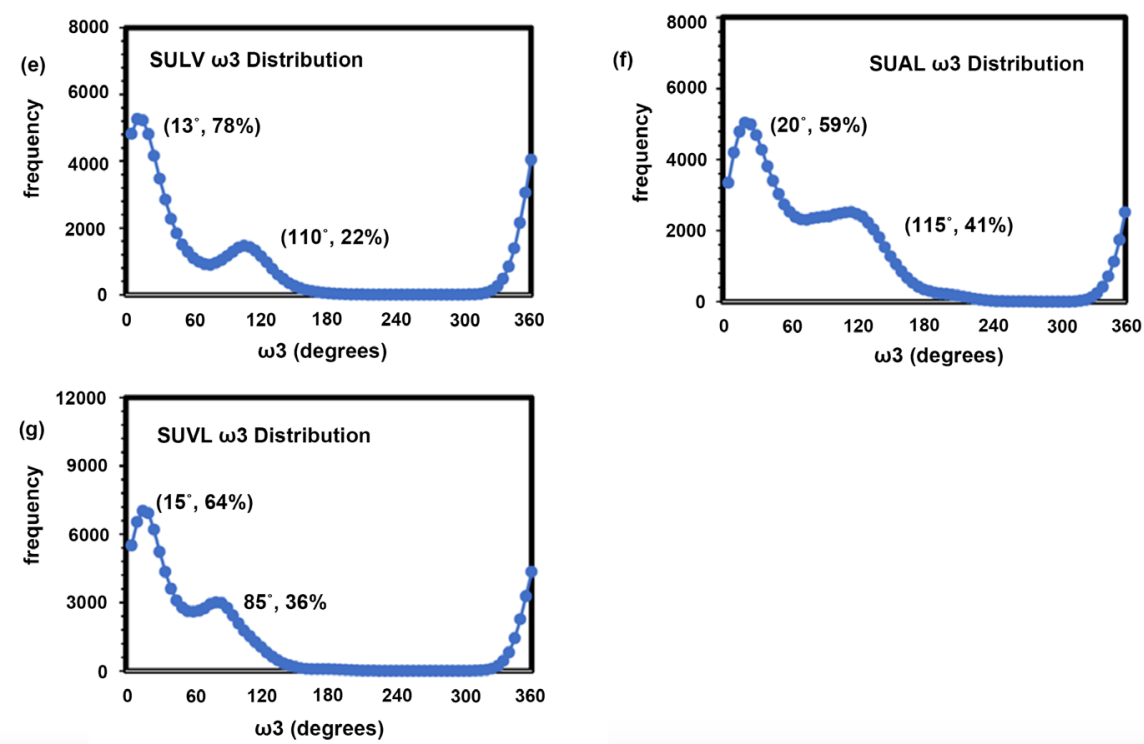

(Part 2)

Supplemental Figure 2. (Part 1) $\omega 3$ dihedral angle distribution histograms for (a) SUVA, (b) SULA, (c) SUAV, (d) SUVV. (Part 2) $\omega 3$ dihedral angle distribution histograms for (a) SULV, (b) SUAL, (c) SUVL. 